\title{
Emergence d'un système d'innovation et de production de biocarburant au Burkina Faso : analyse des déterminants et des enjeux pour son développement
}

\author{
Emergence of a biofuel production and innovation system in Burkina \\ Faso: analysis of the determinants and the stakes for its development
}

\section{Salif Derra ${ }^{1}$}

1UMR Innovation, CIRAD Montpellier, Grdr Bamako, derra_salif@yahoo.fr

\begin{abstract}
RÉSUMÉ. L'émulation née autour de la production des biocarburants a favorisé l'installation de projets biocarburant au Burkina Faso dans l'objectif de promouvoir le développement économique. L'enjeu de cette production a suscité l'installation d'un réseau d'acteurs œuvrant dans sa promotion. Cette étude se propose de caractériser l'émergence du système d'innovation et de production de biocarburant ainsi que les trajectoires de développement observées. L'approche système d'innovation est mobilisée comme cadre d'analyse des conditions de développement de cette innovation. Les résultats soulignent que si les essais de production d'agrocarburants ont été effectués dans les années 80 , le processus d'innovation sur les biocarburants n'a réellement émergé qu'au cours des 5 dernières années. Ils soulignent une faible interaction entre les acteurs mais aussi une méconnaissance des techniques de production. Un vide réglementaire est observé au niveau de ce secteur. L'ensemble de ces défaillances explique la situation toujours en émergence de ce système d'innovation biocarburant.

ABSTRACT. Emulation as a product of biofuel production lead to the installation of biofuel projects in Burkina Faso to promote economic development. The economic potential this production represents has lead to the installation of a network of actors who work towards its promotion. This article aims to characterize the emergence of the production and innovation system of biofuel as well as the development trajectories observed. The innovation system approach is used as a framework for analyzing the development conditions of this innovation. The results point out that while biofuel production tests were conducted in the 1980s, the process of biofuel innovation only really emerged over the past five years. They underline a weak interaction between the actors but also a lack of knowledge of production techniques. A legislative and regulatory vacuum is observed in this sector. All these failures explain the still emerging situation of this biofuel innovation system.

MOTS-CLÉS. Système d'innovation, biocarburant, trajectoire de développement, émergence, Jatropha, déterminants, Burkina Faso.

KEYWORDS. Innovation system, biofuel, development path, emergence, Jatropha, determinants, Burkina Faso.
\end{abstract}

\section{Introduction}

Les crises énergétiques et environnementales engendrées respectivement par la raréfaction des ressources fossiles et les conséquences de leur utilisation, polarisent les recherches technologiques sur la valorisation énergétique de la biomasse agricole. Cet engouement s'est accru suite à la hausse continue du coût de l'énergie mais également avec l'avènement annoncé du pic pétrolier. Les agrocarburants sont alors apparus comme une alternative à explorer dans un contexte de crise de l'énergie (EWING et MSANGI 2009). En outre, ils sont plébiscités comme ayant la capacité de réduire la dépendance énergétique des pays importateurs de pétrole mais également d'améliorer l'accessibilité énergétique des populations marginales (EWING et MSANGI 2009), (BURNOD, GAUTIER et GAZULL 2010).

$\mathrm{Au}$ regard de ces avantages, des projets de production d'agrocarburant se sont développés au Burkina Faso à l'instar des autres pays d'Afrique subsaharienne (ASS). Ces projets ont été initiés par un certain nombre d'acteurs dont l'objectif est de réduire la dépendance énergétique tout en stimulant un développement rural. Dans le cadre de cette production, les acteurs interagissent, s'organisent sous 
forme de réseau en fonction de leur complémentarité autour de cette innovation. L'émergence de ce réseau interpelle sur la nécessité de faire une caractérisation de ce réseau d'acteur afin de procéder à une analyse fonctionnelle. Ce questionnement conduit à analyser le rôle et les fonctions des différentes parties prenantes du réseau d'acteurs ainsi que les institutions qui structurent et activent la production de biocarburant au Burkina Faso. Cette analyse permettra d'esquisser une comparaison entre ce système acteur et le système d'innovation sectoriel dans son acception théorique. Par ailleurs, ce contexte d'émergence et de structuration du réseau d'acteurs, conduit à s'intéresser aux inflexions actuelles de ce système acteur sur la trajectoire de développement des biocarburants au Burkina Faso.

Afin d'apporter un éclairage à ces questionnements, nous présenterons successivement le cadre analytique des systèmes d'innovation sur lequel on s'est appuyé pour cette étude. Ensuite, nous caractériserons le système acteur qui a émergé autour de la production des biocarburants ainsi que les trajectoires de développement impulsées par ce système acteur. Et pour finir, nous discuterons des faiblesses fonctionnelles du système acteur de production de biocarburant.

\section{Approche méthodologique}

\subsection{Cadre analytique}

La production de biocarburant au Burkina Faso est perçue comme une innovation dans le sens où le biocarburant est un nouveau produit. Vue sous cet angle, la production de biocarburant à base de Jatropha $\operatorname{curcas}^{1}$ fait partie de la distinction faite par Schumpeter selon la nature de l'innovation en ces termes :

«L'innovation est une combinaison nouvelle de facteurs de production qui peut s'exprimer par l'introduction de nouveaux produits ; l'introduction de nouvelles manières de production ; l'ouverture de nouveaux marchés et le développement de nouvelles sources d'approvisionnement en matières premières ou en autres intrants ». (SCHUMPETER 1934)

La question de «nouvelle combinaison » ne permet pas de différencier ce qui relève de la créativité et ce qui relève de la mise en œuvre des idées générées, c'est-à-dire de l'innovation (RAHMOUNI et YILDIZOGLU 2011).

L'analyse de l'innovation a beaucoup évolué depuis les travaux fondateurs de Schumpeter. Les nouveaux concepts analytiques de l'innovation n'abordent plus ce phénomène comme un processus invisible de transformation, résultant d'une confrontation statique de l'offre et de la demande, mais elles rendent compte de la nécessité de décrypter tout le processus de la conception à la diffusion. Le point de départ commun à l'ensemble de ces recherches est la considération de l'innovation non plus comme un processus individuel indépendant, mais comme un processus d'interactions entre différents réseaux d'institutions et d'organisations (AMABLE et PETIT 2011). Dans cette optique l'innovation implique nécessairement des interactions entre les acteurs (les firmes, les laboratoires, les universités, etc.) et leur environnement d'où le recours à une approche systémique de l'analyse de l'innovation (système d'innovation).

La notion de « système d'innovation » (SI) rassemble diverses tentatives d'incorporer des éléments institutionnels dans l'analyse économique du changement technique et de la genèse de l'innovation technologique. Considérant le processus d'innovation dans une perspective dynamique, le système d'innovation regroupe un ensemble d'acteurs et d'institutions qui interagissent pour permettre l'utilisation et la création de connaissances.

\footnotetext{
${ }^{1}$ Plante oléagineuse tropicale dont l'huile présente des caractéristiques très proches du diesel
} 
Le concept de système d'innovation est utilisé à l'origine sur une échelle nationale (système national d'innovation) (LUNDVALL 1985). Cette approche originelle s'intéresse à l'analyse du rôle joué par le contexte institutionnel dans la création des conditions favorables à l'innovation et la maîtrise de la technologie. Elle met en exergue les interactions entre les firmes publiques, privées, universités et institutions qui facilitent la production de la science et de la technologie au sein des frontières nationales (environnement de ces acteurs). Par ailleurs, Uzunidis pour sa part, affirme que :

« Un système national d'innovation décrit les relations entre institutions, privées et publiques - scientifiques, technologiques, industrielles, commerciales, financières, politiques - (entreprises, laboratoires de recherche et d'ingénierie, administrations...). Ces relations sont constituées le plus souvent des flux financiers et informationnels et des mouvements de personnes ». (UZUNIDIS 2004)

L'approche système d'innovation connaît des variantes particulières telles que les systèmes régionaux d'innovation (SRI) et les systèmes sectoriels d'innovation (SSI). Le système d'innovation régional (SAXENIAN 1994), appliquent l'approche système d'innovation sur un territoire plus réduit présentant des spécificités territoriales (TOUZARD, TEMPLE, FAURE et TRIOMPHE 2015).

Le système d'innovation sectoriel cherche à mettre en évidence l'importance des éléments propres à un secteur d'activité en mettant en exergue les interactions entre firmes, centre de recherche et de formation et des institutions gouvernementales qui facilitent la production de connaissances et de technologies au sein d'un secteur de l'économie d'un pays (BRESCHI et MALERBA 1997). L'innovation s'effectue dans différents environnements sectoriels. Le secteur est un ensemble d'activités unifié par certains groupes de produits liés à une demande donnée ou émergente et caractérisés par un socle commun de connaissances.

Malerba définit le SSI comme étant un ensemble d'agents qui interagissent à travers des relations marchandes et non marchandes pour la création, la production et la vente de nouveaux produits ou services (MALERBA 2002). Ce système sectoriel est constitué d'une base de connaissances, des technologies, des intrants et d'une demande existante ou potentielle. Les agents du système sectoriel d'innovation sont des organisations (universités, les institutions financières, les organismes gouvernementaux, syndicats, associations ou techniques) et des particuliers (les consommateurs, les entrepreneurs ou les scientifiques). Chaque agent est caractérisé par ses processus d'apprentissage, ses compétences, ses croyances, ses objectifs, ses structures organisationnelles et ses comportements.

Les agents interagissent à travers des processus de communication, d'échange (coopération et concurrence); et ces interactions sont encadrées par les institutions (les règles et les règlements) en place. Au fil du temps, ces interactions induisent des changements et des transformations dans le système sectoriel existant par le biais de la co-évolution de leurs divers éléments ; et un nouveau système sectoriel peut émerger.

L'analyse de l'innovation à travers le concept SSI met l'accent sur un concept spécifique de la structure d'un secteur, exprimée en termes de liens entre des agents, des connaissances, des produits et des technologies. Cela implique également qu'un système sectoriel est un résultat émergent de l'interaction et la co-évolution de ses divers éléments.

\subsection{Collecte de données}

Ce présent article a été rédigé grâce à des enquêtes réalisées en 2013 au Burkina Faso. En effet, on a vu se développer dans ce pays comme dans plusieurs pays de l'ASS ; des projets de production de biocarburant. De 2008 à 2012, environ quinze projets de production de biocarburant ont été mis en place. Les déclarations en termes de superficies emblavées en cultures énergétiques par l'ensemble de ces projets s'élèvent à plusieurs milliers d'hectares (86 000 ha). 
Présentés comme une source d'énergie durable pour répondre à la triple crise énergétique, environnementale et économique, les biocarburants ont été soutenus et promus dans leur développement par un document politique favorable aux investissements en matière de biocarburant. Cette politique en faveur du développement des biocarburants spécifie la possibilité des promoteurs d'emblaver des cultures énergétiques jusqu'à 450000 ha sur le territoire national soit environ $5 \%$ de la superficie agricole utile totale du pays. A cet effet, des terres agricoles ont été accordées à certains promoteurs et des instances de gouvernance du secteur des biocarburants ont été créées.

Cette dynamique autour du développement des biocarburants justifie notre intérêt de faire une analyse afin de comprendre l'émergence du processus d'innovation et de production de biocarburants au Burkina Faso.

La démarche méthodologique s'est basée sur des enquêtes et des entretiens semi-directifs auprès des différentes parties prenantes du secteur des biocarburants. Ces enquêtes ont été menées entre 2012 et 2013. De manière générique trois dispositifs d'enquête ont structuré notre recueil de données :

- un premier au niveau auprès des institutions qui élaborent les politiques publiques de recherche et d'innovation;

- un deuxième niveau relatif aux projets de développement qui sont mis en œuvre par différents acteurs dont principalement les entreprises en joint-venture, les ONG et les collectivités territoriales ;

- un troisième niveau qui se polarise sur les acteurs privés à savoir les entreprises et les producteurs des cultures énergétiques.

$\mathrm{Au}$ total 42 interviews ont été réalisées et les données récoltées portent sur l'identification des expériences en matière d'innovation et de production de biocarburant à base de Jatropha au Burkina Faso. En outre, ces entretiens ont été structurés de sorte à mettre en évidence les activités et les interactions qui ont été établies dans le cadre de la production de biocarburant. L'environnement institutionnel ainsi que la dynamique de développement ont également été l'objet d'entretien avec les acteurs.

L'ensemble de ces entretiens va nous permettre de caractériser les différents aspects du réseau que sont : les agents ; les connaissances et processus d'apprentissage ; les technologies du secteur ; les mécanismes de coordination et les institutions qui encadrent le secteur de biocarburants.

\section{Caractérisation du système d'innovation et de production de biocarburant}

L'émulation née autour de la production des agrocarburants a permis l'émergence de promoteurs de projet, d'organes de recherche dédiés, de producteurs, etc.... L'enjeu que représente la production de biocarburant suscite l'implication d'organismes tels que les institutions publiques, les institutions internationales, le secteur privé, les ONG, les institutions de recherche, les médias et la société civile. L'articulation entre ces agents constitue le système sectoriel qui œuvre dans l'innovation et la production de biocarburant.

La figure ci-dessous représente l'ensemble des agents et des organisations inter reliés dans des relations marchandes et non marchandes avec comme objectif principal la promotion des biocarburants. 


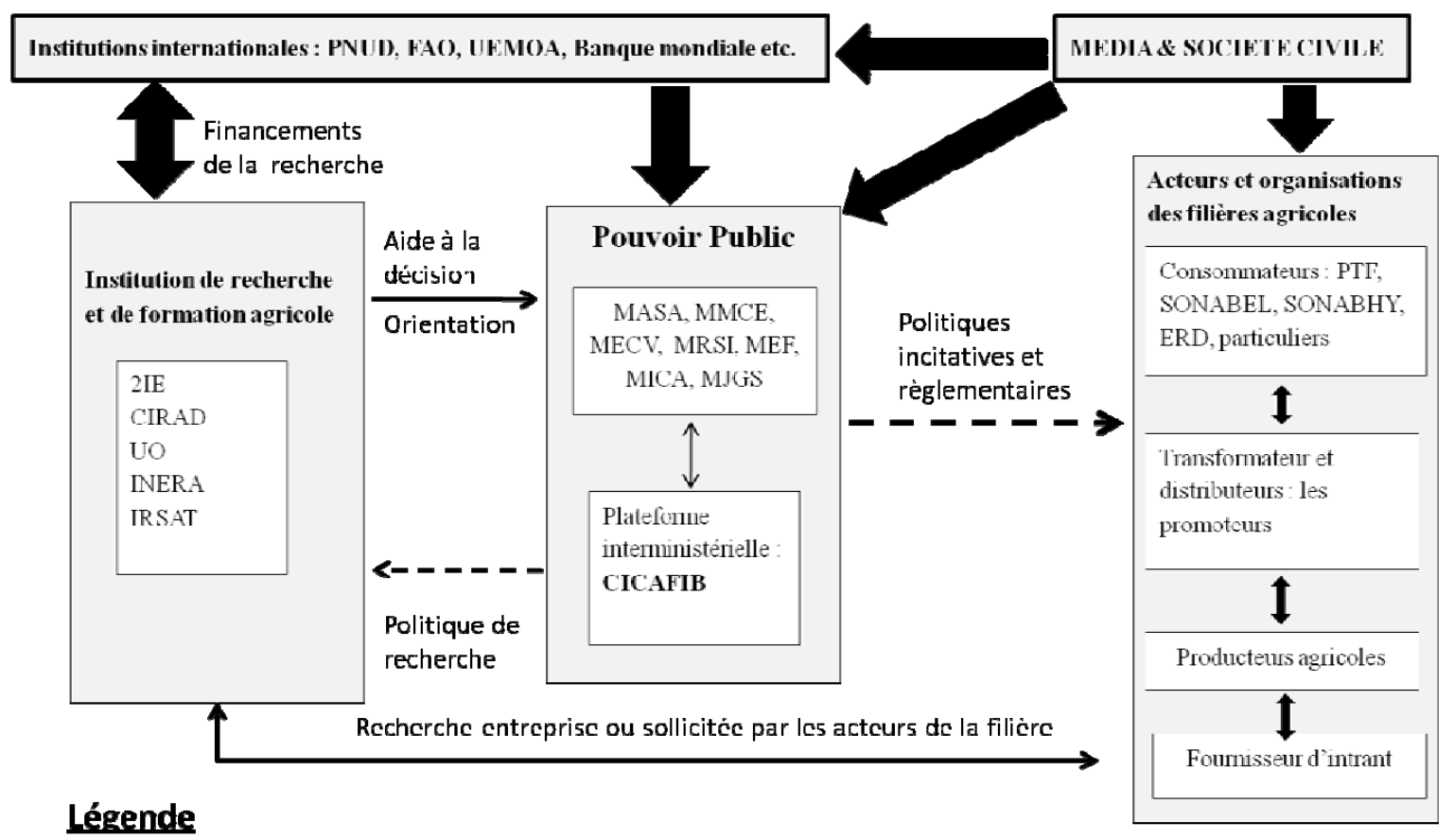

Légende

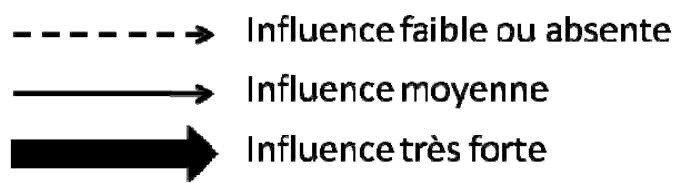

Figure 1. Le système acteur autour de l'innovation et la production de biocarburants au Burkina Faso (Inspiré du schéma conceptuel d'Arnold et Bell 2001)

La figure ci-dessus met en exergue quatre principales composantes du système d'innovation biocarburant ainsi que les interactions existantes entre elles.

- Les institutions de recherche et de formation sur les biocarburants se composent de l'institut d'ingénierie d'eau et d'environnement (2iE), du centre de coopération internationale de recherche agronomique pour le développement (CIRAD), de l'université de Ouagadougou, et du centre national de recherches scientifiques et technologiques (CNRST). Elles entreprennent des relations avec l'ensemble des autres intervenants du système afin de produire de la connaissance nécessaire pour faciliter la mise en œuvre de leurs activités. A cet effet, les acteurs de la chaîne de valeur du système coordonnent leurs besoins en termes de connaissances avec les champs de recherche desdites institutions. En outre, les organes techniques des pouvoir publics mutualisent leurs travaux avec ces instituts de recherche afin de fournir aux organes décisionnels de l'Etat des informations nécessaires pour l'élaboration des cadres réglementaires du secteur des biocarburants.

- Les organisations internationales constituent des éléments majeurs du système d'innovation biocarburant national. A travers leurs financements et leurs orientations politiques, elles influencent de manière forte l'ensemble des parties prenantes du secteur. Il existe un déterminisme fort entre l'émergence du système d'innovation et de production des biocarburants et ces institutions internationales du fait de l'importance de leur pouvoir politique et financier sur l'ensemble des acteurs.

- Les institutions publiques dans le secteur des biocarburants sont chargées de définir le cadre réglementaire et fiscal qui va assurer la sécurité des investissements des acteurs travaillant dans la production, la transformation et la distribution/commercialisation des biocarburants. En outre, elles doivent également mettre en place des normes qui garantissent la qualité des produits au niveau des utilisateurs/consommateurs. Ces institutions publiques sont représentées par le comité interministériel 
chargé de la coordination des activités des filières biocarburants $\left(\mathrm{CICAFIB}^{2}\right)$, les administrations locales et les collectivités territoriales.

- Les acteurs des filières constituent la quatrième composante du système d'innovation qui est chargée d'assurer les principales activités de la filière d'innovation à savoir la production du Jatropha, la transformation du Jatropha en biocarburant et l'utilisation des biocarburants. Ils regroupent les agents économiques qui mobilisent les connaissances codifiées et tacites, les informations, les ressources financières afin de produire et d'utiliser les biocarburants pour la transition énergétique.

Ces différentes composantes s'influencent mutuellement sous forme de financements, directives ou recommandations. Ces relations ont été soulignées par les enquêtes effectuées auprès des acteurs constitutifs de chaque composante. Dans ces enquêtes, il s'agissait d'identifier pour chaque acteur, les initiatives des autres acteurs qui impactent son activité. Ces différentes interactions observées au niveau du système d'innovation a pour objectif de (i) renforcer les capacités et les compétences en matière de biocarburant de chaque agent, (ii) soutenir financièrement et techniquement la création de partenariats novateurs et le développement des biocarburants.

\section{L'environnement incitatif de l'émergence du système acteur}

L'émergence de ce système acteur est le résultat d'une conjugaison de plusieurs facteurs à savoir la mise en place de politiques de soutien aux biocarburants, l'augmentation de la demande mondiale, l'accroissement des financements dans la recherche et la production de biocarburant.

\subsection{Les politiques de soutien aux biocarburants}

La transition énergétique nécessite la mise en place de politiques publiques incitatives (PERCEBOIS 2001), (SORDA, BANSE et KEMFERT 2010). Ces différentes politiques élargissent les opportunités d'investissement pour des acteurs économiques. L'émergence du système acteur autour de la production de biocarburant au Burkina Faso a eu pour précurseur les directives européennes en matière d'énergies renouvelables.

La mise en agenda du problème environnemental a été faite dans de nombreux pays et, en particulier, par l'Union Européenne qui a élaboré en 2007 des politiques publiques incitatives fortes pour le développement des agrocarburants (taux d'incorporation de $10 \%$ en 2020, exonération fiscale, subventions à l'investissement). Cette conjoncture a déclenché des investissements productifs dans les biocarburants en vue de répondre à cette demande. Par ailleurs, le développement des marchés des crédits carbones et, en particulier du marché volontaire, ainsi que les programmes de soutien aux « mécanismes de développement propre » ont également offert aux investisseurs des opportunités de financement ${ }^{3}$ (TSAYEM-DEMAZE 2009). De ce fait, la production mondiale de biocarburants n'a cessé de croître au cours de la dernière décennie, passant de 16 milliards de litres en 2000 à plus de 100 milliards de litres en 2011 (PLATEFORME BIOCARBURANTS 2012).

Les pays du sud présentés comme un espace privilégié de la production de la plupart des cultures énergétiques (conditions climatiques propices, faibles coûts du foncier et de la main-d'œuvre) ont observé l'émergence de plusieurs acteurs œuvrant dans le secteur des biocarburants mais également le développement de plusieurs projets de production (CHERUBINI et STRØMMAN, 2011).

\footnotetext{
${ }^{2}$ Le CICAFIB est constitué de 8 ministères qui sont : le Ministère des Mines, des Carrières et de l'Energie (MMCE); le Ministère de l'Agriculture et Sécurité Alimentaire (MASA) ; le Ministère de l'Economie et des Finances (MEF); le Ministère de l'Environnement et du Cadre de Vie (MECV) ; le Ministère de la Recherche Scientifique et de l'Innovation ; le Ministère du Commerce, de l'Industrie et de l'Artisanat ; le Ministère des Enseignements Secondaire et Supérieur et le Ministère de la Justice et la Garde des Sceaux.

${ }^{3}$ Les projets de production d'agrocarburants peuvent être éligibles en permettant la substitution d'un carburant conventionnel à un carburant « propre » ou, pour les cultures pérennes retenus, en créant des puits de carbone.
} 
Dans le prolongement de l'impulsion des politiques européennes, des politiques régionales africaines et nationales ont été mises en place pour soutenir le développement des biocarburants en ASS. Les institutions régionales telles que l'Union Economique et Monétaire Ouest Africain $\left(\mathrm{UEMOA}^{4}\right)$ ou la Communauté Economique Des Etats de l'Afrique de l'Ouest (CEDEAO) incitent en effet ses pays membres à mettre en place des mesures favorables au développement des biocarburants.

Au niveau du Burkina Faso, une politique visant à favoriser le développement de nouvelles sources d'énergie a été élaborée. Cette politique incitative fait la promotion du potentiel de production de biocarburant, et invite les acteurs économiques à investir dans le secteur des biocarburants. En effet, des actions telles que la cession de terres, la création d'instances de gouvernance sectoriel ont été mises en œuvre afin de faciliter l'essor des biocarburants.

\subsection{Accroissement des financements dans la recherche dédiée aux biocarburants}

Dans la dynamique de développer les biocarburants au sud, des financements ont été consentis dans la recherche et développement de technologies adaptées au niveau des pays du Sud (AMIGUN, MÜLLER-LANGER et VON-BLOTTNITZ 2008). Les politiques publiques en matière de promotion des biocarburants soutiennent des programmes de recherche pour le développement des biocarburants (SMEETS, FAAIJ, LEWANDOWSKI et TURKENBURG 2007). Ces programmes se focalisent sur deux axes.

- En premier lieu, la recherche agronomique dédiée aux cultures énergétiques : le Jatropha, le ricin, le palmier à huile, etc. L'objectif est d'identifier le potentiel productif de ces cultures en fonction des conditions édaphiques et climatiques.

- En second lieu, la recherche technologique sur la production et l'utilisation des biocarburants : la stabilisation des conditions de production et d'utilisation ; l'adaptabilité ou la conception de moteurs fonctionnant aux biocarburants.

Les principaux bailleurs de la recherche sur les biocarburants sont : 1'Union Européenne, les ÉtatsUnis, le Brésil, et des organismes internationaux (Banque Mondiale, FAO, PNUE, ONUDI, etc...).

L'accroissement des financements dans la recherche vise à produire des connaissances mobilisables pour l'implémentation des projets de développement de biocarburant. En Afrique subsaharienne et plus particulièrement au Burkina Faso, les financements au niveau de la recherche se sont focalisés sur la culture du Jatropha Curcas. L'estimation de ces financements faite par les services de la recherche s'élève à plus d'une dizaine de millions d'euro (BOCCANFUSO, COULIBALY, TIMILSINA et SAVARD 2013). En outre, ces financements ont permis la création du laboratoire biomasse énergie et biocarburants (LBEB) au sein de l'institut international d'ingénierie de l'eau et de l'environnement (2iE).

L'ensemble de ces recherches sur la mise au point des technologies de production et d'utilisation des biocarburants a facilité l'émergence des acteurs de la filière d'innovation sur les biocarburants. L'appréhension de ces investissements dans la recherche a stimulé les acteurs économiques à s'investir dans le secteur des biocarburants.

\footnotetext{
${ }^{4}$ UEMOA : Union Economique et Monétaire Ouest Africain compte 8 pays de l'Afrique de l'ouest que sont: le Bénin, le Burkina Faso, la Côte d’Ivoire, la Guinée-Bissau, le Mali, le Niger, le Sénégal et le Togo.
} 


\subsection{Financement des projets de production de biocarburants}

La production de biocarburant comme toute autre filière nécessite un accès aux financements afin d'enclencher le processus de production. L'accès au capital étant un obstacle majeur, plusieurs fonds d'investissement ont été créés afin de soutenir les investissements productifs du secteur des biocarburants. Ces fonds ont servi à financer les activités de la filière biocarburant notamment la production de la matière première et l'installation des unités de transformation. Cette accessibilité au financement a favorisé l'émergence de plusieurs acteurs du secteur. En effet, plusieurs projets de production de biocarburant ont été mis en place. On dénombre une centaine de projets de production de biocarburant en ASS (Fasobiocarburant, Agro-ED, Agritech Faso, Belwet Biocarburant, Ilaria Burkina) émanant d'une cinquantaine d'entreprises étrangères (GRAIN 2012).

Le développement des biocarburants au Burkina Faso à l'instar des autres pays d'ASS s'est amorcé grâce aux financements internationaux. Les différents fonds d'investissement, en plus d'amener les pouvoirs publics burkinabé à mettre en place un cadre incitatif, ont favorisé l'installation des divers agents dans les activités de production des biocarburants. Les bénéficiaires de ces financements sont en général les entreprises promotrices de biocarburant, les centres de recherche et de formation (2iE, universités, centre national de recherche, etc...), les associations (Impulsion, Aprojer), les ONG.

\section{Analyse fonctionnelle du système d'innovation et de production de biocarburant}

\subsection{Création de plateforme pour le renforcement des capacités}

Pour soutenir l'émergence de la production de biocarburant, des actions ont été menées afin de renforcer les capacités des parties prenantes (agricole et industriel). L'objectif est de créer un capital humain doté de compétences et de connaissances nécessaires au développement des biocarburants. La connaissance constitue un élément essentiel dans le démarrage d'une nouvelle activité productive; (LUNDVALL et JOHNSON 1994), (MALERBA 2002).

Les investissements dans le renforcement des compétences et du capital humain se sont matérialisés entre autres par la création d'un laboratoire de recherche sur les bioénergies (LBEB) au sein du $2 \mathrm{iE}$. L'objectif de ce laboratoire est de produire des connaissances pratiques que les acteurs (industriel, utilisateurs) pourraient utiliser pour mener à bien leurs activités dans la filière.

Par ailleurs une conférence biannuelle sur les biocarburants a été instaurée dont la première fut tenue en 2007. La dernière en date (novembre 2013) dont le thème était « les biocarburants : quels potentiels pour l'Afrique » a compté plus de 300 participants constitués de chercheurs, de décideurs politiques et de promoteurs de projet. L'objectif était de discuter des potentialités techniques, économiques, sociales, environnementales des biocarburants pour l'Afrique. Cette conférence est un lieu d'échange et de partage des résultats de recherche sur les biocarburants pour les chercheurs et les porteurs de projet. Elle constitue une base de connaissances sur laquelle les décideurs politiques, et les bailleurs de fonds (UE, Brésil, Taiwan....) vont s'appuyer pour la mise en place de politiques d'encadrement ou de financements. Les interrogations posées généralement dans ces conférences permettent d'avoir une perception sur les conséquences des choix de la filière biocarburant (choix de la culture, type de presse, produit fini etc.).

\subsection{Fonctionnement du secteur des biocarburants au Burkina Faso}

La représentation systémique du secteur des biocarburants permet d'analyser les rôles et le niveau d'influence des agents et organisations dans la définition d'une trajectoire de développement des biocarburants. On entend par trajectoire, l'ensemble des choix et compromis fait tout au long de la filière de production. Ces choix portent sur la culture, la conduite culturale, le matériel industriel pour la trituration et la transformation, l'output... 
Ces choix sont faits par les agents de la chaîne de valeur et plus particulièrement les promoteurs de projet de production. Au nombre de 12, ces promoteurs s'appuyant sur les résultats produits par les institutions de recherche et de formation mais aussi sur les institutions en vigueur pour leur choix. L'implémentation des modèles de production du secteur s'observe à leur niveau.

Le domaine de la recherche constitue le précurseur de la trajectoire de développement grâce à leurs résultats. Ces résultats sont utilisés par les acteurs de la chaîne de valeur et aussi par les organes de régulation tels que la CICAFIB pour orienter le développement souhaité par l'Etat. En effet, la CICAFIB impose par exemple aux porteurs de projet de production de biocarburant un régime de déclaration des superficies cultivées dans leurs zones d'action. Le but est de s'assurer que l'ensemble des superficies occupées par les cultures énergétiques n'excède le plafond fixé soit $5 \%$ de la surface agricole utiles du pays (450 $000 \mathrm{ha})$.

Par ailleurs, une partition importance dans la définition de la trajectoire de développement est jouée par la coopération internationale. Les agents de la chaine de valeur mais aussi des autres volets du système d'innovation et production bénéficient d'aides financières et de soutiens logistiques. La plupart des projets de production en cours ont des financements extérieurs. Certains ont même bénéficié de soutiens logistiques grâce à la coopération qu'entretient l'Etat burkinabé avec Taiwan. L'ensemble de ces accompagnements oriente d'une certaine manière l'évolution du secteur. En outre, les institutions régionales telles que l'UEMOA et la CEDEAO octroient des financements dans le but d'impulser l'émergence d'une politique d'encadrement commune entre les pays membres.

\subsection{Modèles de développement des biocarburants}

Deux modèles de développement du biocarburant à base de Jatropha curcas émergent du système d'innovation biocarburant. Il s'agit du modèle dit « circuit court » et du modèle agro-industriel.

- La trajectoire «bioénergie pour l'électrification rurale» souvent appelée «circuit court » qui caractérise un modèle de développement dans lequel les phases de production - transformation et utilisation sont spatialement intégrées c'est-à-dire concentrées dans la même localité. Les projets qui entre dans cette catégorie présentent des unités de production moins capitalistique $(1$ hectare au maximum par producteur de matière première, unité de transformation de petite capacité 60 à $100 \mathrm{~kg} /$ heure, etc.). Ces types de projet sont mis en œuvre ou soutenus par des organismes de soutien au développement dans l'optique d'augmenter l'accessibilité et la disponibilité des services énergétiques dans les zones rurales. La faible dimension des unités de production notamment l'unité de transformation pose la question de la durabilité de cette trajectoire du fait de sa rentabilité (AMIGUN, SIGAMONEY et VON-BLOTTNITZ 2008). En effet, cette trajectoire présente de faibles rendements d'échelle au niveau de la production mais également une qualité variable du biocarburant (PORTALE 2012).

- La trajectoire «biocarburant pour le transport» également appelée «modèle industriel» se caractérise par un dispatching géographique des phases de production-transformation-utilisation. Le principe de fonctionnement de cette trajectoire s'appuie sur le modèle de l'industrie agroalimentaire. La production de matière première est faite en zone rurale, la transformation et l'utilisation en majorité se font en milieu urbain. Etant donné, la taille du marché à couvrir (nationale voire international), les unités de transformation présentent de grandes capacités faisant ainsi bénéficier de rendements d'échelle. Les promoteurs référenciés dans cette catégorie sont des grandes entreprises souvent filiales de groupes internationaux. Les avantages de tel modèle reposent sur sa productivité. En effet, les unités de transformation étant de grandes capacités arrivent à générer des économies d'échelle sous réserve de disponibilité suffisante de matière première. Cette trajectoire soulève des controverses en raison des risques de concurrence qu'elle pose par rapport aux productions alimentaires (EWING et MSANGI 2009), (EIJCK, SMEETS et FAAIJ 2012), (TEMPLE, TOUZARD, KWA, BOYER et REQUIER DESJARDINS 2015). Fondée sur une production à grande échelle, l'allocation des terres pour la 
production de matière première présente des risques de précarisation de la sécurité alimentaire dans le contexte d'accroissement démographique.

\section{Les défaillances du système d'innovation et de production de biocarburant}

Bien que l'idée de produire du biocarburant ait émergé depuis 2007, la filière jatropha curcas est toujours en phase de construction. Le constat est qu'il existe toujours un gap profond entre l'idée de production et les changements implicites et investissements qu'il faut pour réellement produire. Pourtant, il reste indéniable qu'il existe au Burkina Faso des potentialités agronomiques propices pour la production des agrocarburants. Malgré la mise en place de projets de production et des institutions de promotion, on constate des conditions défavorables à l'essor du secteur. Ces goulots d'étranglement concernent la méconnaissance des conséquences des choix technologiques, la faiblesse des interactions entre les agents du secteur et le manque de cadre règlementaire et incitatif.

\subsection{Insuffisance de la connaissance sur les conséquences des choix technologiques}

Impulsée par des entreprises étrangères ou par des ONG, la culture du jatropha curcas s'est faite sans réelle connaissance au préalable du savoir-faire et des besoins en investissement (temps, capital). Chaque promoteur en fonction de ses connaissances préalables recommande un mode de conduite culturale. On rencontre de ce fait autant d'itinéraires techniques que de promoteurs. Le manque d'harmonisation entraine un flou au niveau des agriculteurs qui ne savent plus laquelle des recommandations conduit à de bon rendement. Par ailleurs du côté de la transformation, il y a aussi un manque de standards ou de normes de qualité de l'huile végétale carburant. Ceci explique la coexistence de deux méthodes de pressage : pressage avec ou sans torréfaction. Le taux d'extraction et la qualité de l'huile varie selon la méthode utilisée (VAITILINGOM 2007).

\subsection{Faiblesse des interactions au sein du système acteur}

Une des conditions critiques observée au niveau de la filière biocarburant est la faiblesse des interactions entre les agents. L'absence d'harmonie dans les choix technologiques tout le long de la filière biocarburant résulte de la faiblesse des interactions entre les acteurs. Hormis la tenue bisannuelle de la conférence biocarburant qui ne regroupe d'ailleurs que les décideurs, chercheurs et promoteurs, il n'existe pas d'autre cadre formel d'échange et de collaboration entre les acteurs. Il manque une véritable interaction entre chercheurs, innovateurs, décideurs politiques, producteurs, transformateurs, commerçants, consommateurs, médias, financiers, etc... pouvant permettre des synergies d'action afin de promouvoir les innovations dans la filière. Les sphères du système national d'innovation demeurent faiblement intégrées les unes aux autres. L'isolation des acteurs économiques apparaît aussi comme une raison primordiale de la difficulté à construire des capacités technologiques nationales.

Du coté des pouvoirs publics, il y a une faible coordination des institutions publiques dans l'encadrement du développement de la filière. Cela se traduit entre autre par des directives souvent contradictoire au niveau du terrain. Le ministère de l'environnement devant être au cœur de l'activité se trouve en second plan et ne donne aucune directive en matière de production du Jatropha. Les rares initiatives paysannes qui ont bénéficiées d'un appui proviennent du ministère de l'agriculture. Il s'agissait d'un accompagnement des producteurs pour la mise en place de groupement de producteurs de Jatropha. Elles ont été d'ailleurs stoppées du fait que le jatropha étant une plante forestière, sa promotion relève des services de l'environnement.

Du côté de la production et de la transformation, on constate une faible organisation. Chaque promoteur agit de son côté. Il y a un esprit de méfiance et de concurrence entre les promoteurs de biocarburant. Cette situation décrédibilise les promoteurs vis-à-vis des services déconcentrés de l'Etat et des producteurs. 


\subsection{Manque de cadres réglementaires et de normes}

La stratégie du Burkina Faso en matière de biocarburant est axée sur une régulation de l'exploitation du Jatropha curcas. Elle cherche à encadrer l'engouement qui a conduit à planter du jatropha sans aucune visibilité sur la qualité du produit, ses implications et ses coûts. Le document cadre qui marque la volonté de l'Etat à s'engager dans le développement des biocarburants n'est pas clairement formalisé comme une stratégie. Le document " politique cadre sur les biocarburants » évoque trois conditionnalités (adéquation avec la sécurité alimentaire ; protection de l'environnement, de la biodiversité et du développement durable ; respect de la paysannerie traditionnelle) à respecter pour tout projet biocarburant tout en mettant l'accent uniquement sur le plafonnement des surfaces à allouer aux cultures énergétiques. Aucun objectif quantifié n'est défini, malgré l'existence du comité interministériel ; il n'existe pas de normes sur les biocarburants. Par ailleurs, il y a une faible coordination des activités d'appui des institutions publiques du secteur (GATETE et DABAT 2014). Cette insuffisance de coordination entraine souvent des divergences qui sont source de conflit au détriment de la coopération et de la synergie d'action.

\subsection{Interpellations de la société civile au niveau national et international}

La production de Jatropha curcas se faisant pas dans des zones arides, constitue une source de compétition sur la production vivrière. Cette compétition porte sur l'utilisation des facteurs de production, notamment la terre, les ressources en eau, la force de travail et les fertilisants nécessaires (FAO, 2009). Par ailleurs, un certain nombre de travaux tendent de plus en plus à contester la pertinence du développement des biocarburants au regard de leur instrumentalisation par les entreprises internationales qui passent par les élites public, pour s'accaparer des surfaces de terres importantes (COTULA, DYER et VERMEULEN 2008), (SULLE et NELSON 2009). Les conséquences réelles du développement des biocarburants sur la sécurité alimentaire restent pour l'instant encore difficiles à appréhender au regard des données mobilisables. Elles interrogent la capacité des cadres méthodologiques d'évaluation d'impact de la recherche à rendre compte de ces causalités (TEMPLE, BIÉNABE, BARRET et SAINT-MARTIN 2016). Dans les conditions actuelles, elles sont peu perceptibles mais ces conditions renvoient à une situation où la production de jatropha est encore marginale et largement insuffisante pour rendre rentable l'industrialisation nécessaire à la production d'une énergie compétitive par rapport aux énergies fossiles (AMIGUN, MÜLLER-LANGER et VONBLOTTNITZ 2008).

\section{Conclusion}

Le Burkina Faso à l'instar d'autres pays d'Afrique subsahariens a entrepris la production d'agrocarburants en vue de répondre à des incitations financières internationales et des enjeux économiques et environnementaux. Pour répondre à ces incitations et enjeux, plusieurs agents et organisation se sont mobilisés. Il s'agit des institutions publiques ; des entreprises privées, des ONG ; des centres de recherche et universités, des agriculteurs ; de la société civile et des médias. L'ensemble de ces acteurs et organisations œuvrant dans la promotion des biocarburants constitue une structure que l'on peut analyser à travers le concept de système sectoriel d'innovation et de production de biocarburants. Ce «système » propose de répondre à la forte demande d'agrocarburants au niveau international ; aux opportunités offertes par les investissements de recherche internationaux publics et privés; et aux impulsions politiques nationales en relation avec des demandes d'accès à l'énergie dans certaines zones localisées.

Après caractérisation du système sectoriel à travers la mise en évidence du rôle et les interactions des agents, l'analyse de ce dernier montre que le secteur privé notamment les promoteurs financés par des multinationales pilotent les choix technologiques. Les orientations concernant les biocarburants à base du Jatropha curcas se déclinent en deux modèles de développement. Il s'agit d'un part, du modèle de production en circuit court destinée à fournir des services énergétiques en milieu rurale et 
d'autre part du modèle agro-industriel pour la production de biodiesel pour le secteur des transports. L'analyse du fonctionnement du système sectoriel d'innovation montre des insuffisances telles que la faiblesse des interactions entre les acteurs et l'absence de politiques incitatives encadrant le secteur des biocarburants. Les cadres de concertation existants ne sont pas suffisamment fonctionnels et dynamiques pour favoriser l'interaction des acteurs.

Au-delà de ces insuffisances, le concept de système d'innovation permet de montrer l'incomplétude systémique dans le sens où certaines interactions sont inefficientes ou simplement inexistantes. Les différentes sphères du système d'innovation biocarburant demeurent faiblement intégrées les unes aux autres. Cette utilisation de l'approche système d'innovation permet de comprendre comment les dynamiques d'innovations sur les biocarburants sont gouvernées par l'hybridation entre des cadres institutionnels, financiers technologique globalisés et des dynamiques nationale. Elle souligne dans ce contexte de nombreuses rigidités organisationnelles et institutionnelles liées au développement du secteur.

\section{Bibliographie}

AMABLE B., PETIT P., « La diversité des systèmes sociaux d'innovation et de production dans les années 90 », dans J.P. TOUFFUT (dir.), Institutions et innovation. De la recherche aux systèmes sociaux d'innovation, Éditions Albin Michel, Paris, 2001.

AMIGUN B., MÜLLER-LANGER F., VON BLOTTNITZ H., « Predicting the costs of biodiesel production in Africa: learning from Germany », Energy for Sustainable Development, vol. 12, n¹, pp. 5-21, 2008.

AMIGUN B., SIGAMONEY R., VON BLOTTNITZ H., « Commercialization of biofuel industry in Africa: A review », Renewable and Sustainable Energy Reviews, vol. 12, n³, pp. 690-711, 2008.

BOCCANFUSO D., COULIBALY M., TIMILSINA G., R., SAVARD L., Macroeconomic and distributional impacts of jatropha-based biodiesel in Mali, The World Bank Edition, Washington D.C., 2013.

BRESCHI S., MALERBA F., « Sectoral innovation systems: technological regimes, Schumpeterian dynamics and spatial boundaries », dans C., EDQUIST (dir.), Systems of Innovation, Frances Printer Edition, London, 1997.

BURNOD P., GAUTIER D., GAZULL L., «Les agrocarburants au Mali : nouveau produit, vielles recettes ? Une analyse de l'émergence et des enjeux du système d'innovation agrocarburant» », Innovation and Sustainable Development in Agriculture and Food, France, 2010.

CHERUBINI F., STRØMMAN A., H., " Life cycle assessment of bioenergy systems: state of the art and future challenges », Bioresource technology, n¹02, pp. 437-451, 2011.

COTULA L., DYER N., VERMEULEN S., Fuelling Exclusion? The Biofuels Boom and Poor People's Access to Land, Edition IIED, London, 2008

EIJCK J., SMEETS E., FAAIJ A., « The economic performance of Jatropha, Cassava and Eucalyptus production systems for energy in an East African smallholder setting », GCB Bioenergy, vol. 4, nº6, pp. 828-845, 2012

EWING M., MSANGI S., «Biofuels production in developing countries: assessing tradeoffs in welfare and food security », Environmental Science \& Policy, vol. 12, n, pp. 520-528, 2009.

FAO, Making sustainable biofuels work for smallholder farmers and rural households: Issue and perspectives, Energy and Agriculture Edition, United Nations, Rome, 2009.

GATETE C., DABAT M., H., « Développement des agrocarburants en Afrique de l'ouest, une analyse institutionnelle comparative » Économie rurale. Agricultures, alimentations, territoires, n³44, pp 9-27, 2014.

GRAIN, Panorama: qui est derrière l'accaparement des terres? Regard sur quelques-uns des investisseurs responsables d'acquisitions massives de terres et ceux qui les soutiennent, Edition le GRAIN, Rapport nº38, Barcelone, 2012.

LUNDVALL B.-A., « Product Innovation and User-Producer Interaction », Series Industrial Development Research n³1, Aalborg University Press, Danemark, 1985.

LUNDVALL B.-Ä., JOHNSON B., « The learning economy », Journal of industry studies, vol. 1, n², pp. 23-42, 1994.

MALERBA F., « Sectoral systems of innovation and production », Research Policy, vol. 31, n², pp. 247-264, 2002. 
PERCEBOIS J., « Energie et théorie économique : un survol », Revue d'économie politique, vol. 111, nº, pp 815-860, 2001.

PLATEFORME BIOCARBURANTS, Production de biocarburants dans le monde en 2011, www.plateformebiocarburants.ch/infos/production.php, 2012

PORTALE E., «Socio-economic sustainability of biofuel production in sub-Saharan Africa: evidence from a Jatropha outgrower model in rural Tanzania », Environment and Natural Resources Program Discussion Paper Series n²01201, Cambridge, 2012.

RAHMOUNI M., YILDIZOGLU M., Motivations et déterminants de l'innovation technologique: Un survol des théories modernes, Universités d'Aix Marseille II et III, GREQAM no2011-09, 2011.

SAXENIAN A., « Regional advantages », Havard University Press, Cambridge, 1994.

SCHUMPETER J.A., The theory of economic development: An inquiry into profits, capital, credit, interest, and the business cycle, University of Illinois at Urbana, Historical Research Reference in Entrepreneurship, 1934.

SMEETS E.M., FAAIJ A.P., LEWANDOWSKI I.M., TURKENBURG W.C., « A bottom-up assessment and review of global bio-energy potentials to $2050 »$, Progress in Energy and combustion science, vol. 33, n 1, pp. 56-106, 2007.

SORDA G., BANSE, M., KEMFERT C., « An overview of biofuel policies across the world », Energy Policy, Vol 38, $\mathrm{n}^{\circ} 11, \mathrm{pp}$ 6977-6988, 2010.

SULLE E., NELSON F., Biofuels, land access and rural livelihoods in Tanzania, Russell Press, IIED Edition, London, 2009.

TEMPLE L., BIÉNABE E., BARRET D., SAINT-MARTIN G., « Methods for assessing the impact of research on innovation and development in the agriculture and food sectors. African Journal of Science, Technology, Innovation and Development, 8(5-6), 399-410, 2016. http://dx.doi.org/10.1080/20421338.2016.1219484

TEMPLE L., TOUZARD J.M., KWA M., BOYER J., REQUIER DESJARDINS D., " Comparaison des trajectoires d'innovation pour la sécurisation alimentaire des pays du Sud » Revue Biotechnologie, Agronomie, Société et Environnement, vol 19, n¹, pp. 53-61, 2015.

TOUZARD J.M., TEMPLE L., FAURE G., TRIOMPHE B., «Innovation systems and knowledge communities in the agriculture and agrifood sector: a literature review », Journal of Innovation Economics and Management, vol $2 \mathrm{n}^{\circ} 17$, pp.117-142, 2015.

TSAYEM DEMAZE M., «Paradoxes conceptuels du développement durable et nouvelles initiatives de coopération Nord-Sud : le mécanisme pour un développement propre », Revue Européenne de Géographie. n443, 2009.

UZUNIDIS D., L'innovation et l'économie contemporaine: espace cognitifs et territoriaux, Edition De Boeck, Bruxelles, 2004.

VAITILINGOM G., Extraction, conditionnement et utilisation des huiles végétales pures carburant, Conférence international « Enjeux et Perspectives des Biocarburants pour l'Afrique », Ouagadougou, pp. 54, 2007. 